\title{
The role of cholesterol efflux in regulating the fertilization potential of mammalian spermatozoa
}

\author{
Alexander J. Travis ${ }^{1}$ and Gregory S. Kopf ${ }^{2}$ \\ ${ }^{1}$ Center for Research on Reproduction and Women's Health, University of Pennsylvania School of Medicine, \\ Philadelphia, Pennsylvania, USA \\ ${ }^{2}$ Women's Health Research Institute, Wyeth Research, Collegeville, Pennsylvania, USA \\ J. Clin. Invest. 110:731-736 (2002). doi:10.1172/JCI200216392.
}

Following spermatogenesis and spermiogenesis, mammalian spermatozoa leaving the testis appear to be morphologically mature but clearly are immature from a functional standpoint; that is, they have acquired neither progressive motility nor the ability to fertilize a metaphase II-arrested egg. Although progressive motility is acquired and signaling pathways mature during sperm transit through the epididymis, complete fertilization capacity in vivo is conferred only during residence in the female reproductive tract. Similar observations have been made using a variety of in vitro assays, suggesting that a series of events, some initiated by environmental cues, confer on sperm the ability to fertilize the egg.

This acquired capacity to fertilize was first observed by Austin (1) and Chang (2), who demonstrated that freshly ejaculated sperm cannot fertilize eggs until they reside in the female reproductive tract for a finite period of time. All of the cellular events that allow the ejaculated sperm to fertilize an egg were subsumed into a single phenomenon that was termed "capacitation." The ability to capacitate sperm in vitro has been of great importance to both scientists and clinicians, who have used it to study the basic biology of fertilization and to develop various assisted reproductive technologies for humans and other species. Work by many investigators has established that the process of fertilization, not surprisingly, represents a series of elegant intercellular communication and cellular activation events (3-5). Sperm functions such as motility and capacitation in the female reproductive tract are likely modulated by environmental cues in the luminal fluid, as well as by interactions with oviductal epithelium or other female tissues (6). When sperm

\footnotetext{
Address correspondence to: Gregory S. Kopf, Women's Health Research Institute, Wyeth Research, PO Box 8299, Philadelphia, Pennsylvania 19101-8299, USA. Phone: (484) 865-7846;

Fax: (484) 865-9367; E-mail: kopfg@wyeth.com.

Alexander J. Travis's present address is: The James A. Baker Institute for Animal Health, College of Veterinary Medicine, Cornell University, Ithaca, New York, USA

Conflict of interest: No conflict of interest has been declared. Nonstandard abbreviations used: filipin-sterol complex (FSC); protein kinase $\mathrm{A}(\mathrm{PKA})$.
}

arrive in the oviduct and encounter the ovulated, metaphase II-arrested egg enclosed in its cumulus cell matrix, a complex series of cell-cell and cell-ECM interactions ensues, initiating cellular signaling events that permit the fusion of the sperm and egg plasma membranes. Several of these cell-matrix and cell-cell interactions involve novel gamete surface proteins and matrices. Signal transduction events leading to gamete activation, in particular sperm acrosomal exocytosis and egg cortical granule secretion, share some features with signaling events described in somatic cells.

Sperm membrane cholesterol efflux contributes to one such novel signaling mechanism that controls sperm capacitation, and the details of this effect are now beginning to be understood at the molecular level. Knowledge of how cholesterol efflux occurs in these cells, as well as how this efflux is integrated with transmembrane signaling to regulate sperm function, may reveal much about the fertilization process and may also provide insights into the role and dynamics of membrane cholesterol efflux in somatic cell function. Here, we offer a short overview of the role of cholesterol efflux in regulating sperm capacitation, with an aim toward identifying areas of future investigation that may ultimately provide a greater understanding of the role of this sterol in regulating signal transduction. The reader is referred to several extensive reviews of this subject $(7,8)$.

\section{Molecular basis of capacitation}

After attaining morphological maturity in the testis, sperm must undergo two distinct processes of functional maturation to be able to fertilize an egg. The first occurs in the epididymis of the male reproductive tract, as sperm move from the caput to the corpus and then to the caudal regions of this organ, where they are stored prior to ejaculation. During this transit, the signaling pathways that regulate capacitation are enabled. Thus, caput epididymal sperm fail to be capacitated in the presence of molecular stimuli (defined below) that are sufficient to capacitate sperm residing in the cauda epididymis (9).

Several molecular events are likely involved in the acquisition of signaling competence. For example, concomitant with the maturation of these signaling pathways, 

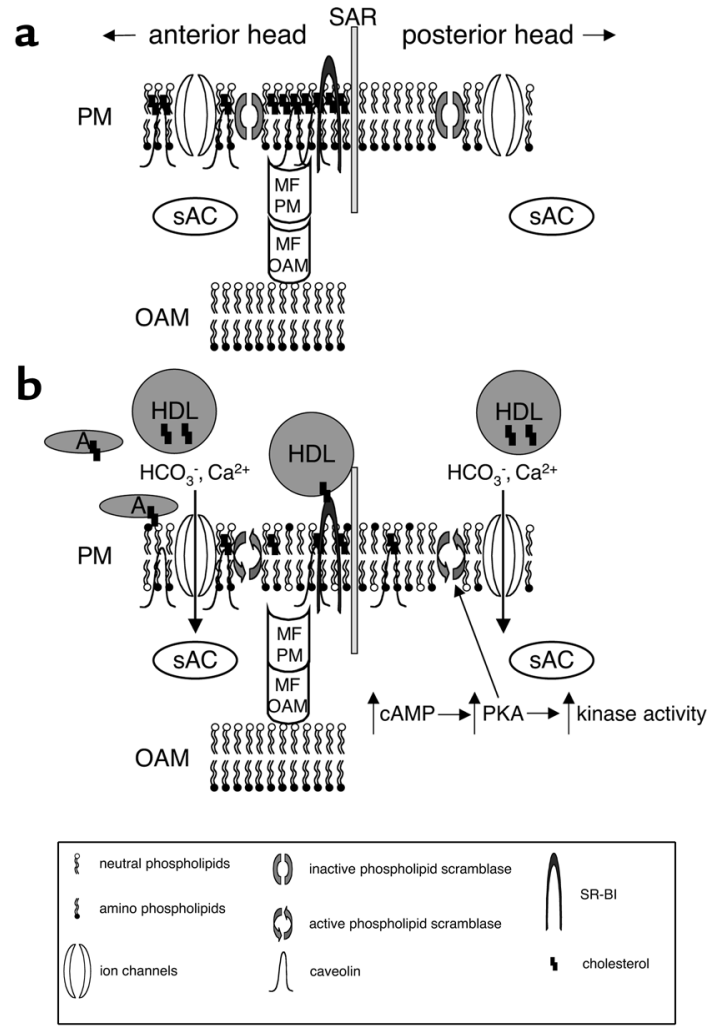

\section{Figure 1}

Model for the organization of cholesterol in the membranes of the sperm head, and its role in signaling. Schematic diagrams of a murine sperm incubated under noncapacitating conditions (a) and capacitating conditions (b). In this model, membrane rafts (regions enriched in sterols) are organized in the plasma membrane of the anterior head. Membrane fusion proteins are inactive due to being tethered by caveolin. The distribution of phospholipids in the membrane leaflets is asymmetrical, as the scramblases are inactive. When exposed to capacitating conditions (bicarbonate, calcium, albumin, and HDLs), cholesterol is removed via a specific pathway (HDL-SR-BI) and a nonspecific pathway (albumin). Increases in SAC activity elevate CAMP levels and PKA activity and stimulate downstream kinases. This signaling results in increased phospholipid scrambling, causing a disordered distribution of amino- and neutral phospholipids. Together with the increase in membrane fluidity caused by the sterol efflux, this change results in lateral movement of some sterols and caveolin from the anterior to the posterior head. The loss of sterols from the membrane causes a disruption of the interaction between caveolin and the membrane fusion proteins, resulting in their activation. The plasma membrane (PM) and outer acrosomal membrane (OAM) are shown immediately adjacent to the subacrosomal ring (SAR). For simplicity, both an anion and a cation are drawn as passing through a single ion channel in $\mathbf{b}$. Cholesterol can exist in a free state in the membrane or be associated with cholesterol acceptors such as HDLs or albumin (A). SAC, a novel $\mathrm{HCO}_{3}{ }^{-}$-activated adenylyl cyclase that associates at least in part with membranes, is shown here in the sperm cytoplasm. Membrane fusion proteins (MF) can associate with either the PM or the OAM.

epididymal sperm undergo dramatic alterations in their membrane sterol content. Such changes are highly species-specific and are also highly specific with regard to the class of sterol that is being changed (10). In addition, intracellular signaling systems that control capacitation mature during epididymal transit (ref. 11; M. Fornes et al. unpublished data). How alterations in membrane sterol composition integrate with the maturation of signaling pathways is still not fully understood.

The majority of alterations of epididymal sperm sterol content probably result from interactions of the sperm with the epididymal epithelium. Epithelial linings of both the epididymis and the vas deferens appear to have a highly developed sterol-producing capacity (12), although the impact of sterol synthetic capacity in the vas deferens on sterol levels in ejaculated sperm is unclear. There are also changes in the content of other sperm lipids during epididymal maturation. In some species, phospholipids are the major source of energy for endogenous oxidative respiration and therefore phospholipid levels decline during epididymal maturation (13). Changes in either sperm sterol or phospholipid levels might serve to alter the membrane cholesterol/phospholipid molar ratio, which has been implicated in the regulation of capacitation, as described below.

Given the species-specific nature of these large-scale alterations in lipid content, it is difficult to speak generally about their function. However, in all species examined thus far, cauda epididymal sperm possess clearly delineated membrane domains that differ in their sterol composition. Initially characterized by the presence of filipin-sterol complexes (FSCs) visible by freeze-fracture electron microscopy, these domains impart heterogeneity on the sperm surface within a given region of these cells. Such subdomains suggest the possibility of still more precise compartmentalization of function beyond the obvious polarization of these cells into head and tail domains that contribute to egg interaction and motility regulation, respectively. Indeed, these subdomains have recently been hypothesized to act as scaffolds or foci for signaling pathways regulating sperm capacitation in both the head and flagellum (14).

\section{Signaling and the control of fertilization competence in sperm}

Recent studies by several laboratories using in vitro models support the idea that capacitation requires transmembrane signaling and intracellular signal transduction. The development of in vitro capacitation protocols for sperm of several different species has shown the critical importance of three media constituents, namely $\mathrm{Ca}^{2+}, \mathrm{HCO}_{3}^{-}$, and a protein that can function as a cholesterol acceptor, such as serum albumin.

Work by several laboratories shows that capacitation is likely regulated by a novel signal transduction pathway involving cross-talk between cAMP, protein kinase A (PKA), and tyrosine kinases (Figure 1). This signaling leads to the phosphorylation on tyrosine residues of several proteins, the identities of which are only starting to be elucidated (7). As initially demonstrated by Visconti et al. (11), cauda epididymal mouse sperm, when incubated in vitro in media known to support capacitation, display a time-dependent increase in protein tyrosine phosphorylation that correlates with the onset of functional capacitation, operationally defined by the ability of the sperm population to fertilize eggs. The apparent absence of external stimuli, such as hormones or cytokines, that might initiate the observed tyrosine 
phosphorylation suggested that signaling might be regulated by a time-dependent mechanism or by specific components in the media. Subsequent work showed that the extracellular $\mathrm{Ca}^{2+}, \mathrm{HCO}_{3}^{-}$, and serum albumin in the capacitation medium are all absolutely required for these molecular and functional changes (11) and implicated a novel adenylyl cyclase/cAMP/PKA signaling system in sperm capacitation (7).

Just as interesting as this novel mode of signal transduction is the unusual mechanism by which these medium components activate cAMP signaling (Figure 1). Although there is clear evidence that $\mathrm{Ca}^{2+}$ can regulate the activity of specific adenylyl cyclase and cyclic nucleotide phosphodiesterase family members, the effects of $\mathrm{HCO}_{3}^{-}$on adenylyl cyclase activity have been demonstrated in only a small number of cells or tissues, including ocular ciliary processes, corneal endothelium, choroid plexus, the medullary and cortical regions of the kidney (15), and sperm (4). Presently, most is known about the sperm adenylyl cyclase. This enzyme is not regulated in a manner similar to that seen with the classical 12-transmembrane, $G$ protein-regulated somatic cell adenylyl cyclases (4). A considerable amount of effort has been devoted to characterizing the sperm $\mathrm{HCO}_{3}{ }^{-}$-activated adenylyl cyclase, which was recently purified and cloned (16). This protein, now termed "sAC," has many novel characteristics and is likely to exist in multiple forms as a consequence of alternative splicing and proteolysis. Its catalytic domains resemble the adenylyl cyclases of Cyanobacteria, enzymes that can also be regulated by $\mathrm{HCO}_{3}^{-}(17,18)$.

\section{Role of cholesterol efflux in signal transduction leading to capacitation}

The historical requirement for serum albumin in defined media to support capacitation had been hypothesized by several groups to be due to the ability of albumin to serve as a sink for cholesterol removal from the sperm plasma membrane $(10,19)$. Removal of this sterol likely accounts for the changes in membrane fluidity observed during capacitation and the subsequent decrease in the membrane cholesterol/phospholipid ratios (7). Such changes in membrane dynamics are likely to significantly affect cellular function (see below). Coupled with evidence that sperm membrane sterol levels decline following exposure to albumin, the observation that other cholesterol acceptors like HDL and $\beta$-cyclodextrins effectively support capacitation suggests that the primary action of serum albumin is in mediating cholesterol efflux $(20,21)$. Interestingly, the action of serum albumin, HDL, and $\beta$-cyclodextrins as cholesterol acceptors is somehow coupled to the cAMPdependent pathway described above (Figure 1) $(20,21)$. How might changes in the sterol content of the membrane regulate transmembrane signaling leading to capacitation? Biophysical studies demonstrate that cholesterol alters the bulk properties of biological membranes. For example, this sterol can increase the orientation order of the membrane lipid hydrocarbon chains, restricting the ability of membrane proteins to undergo conformational changes by rendering their surrounding membrane less fluid. High concentrations of cholesterol can thereby inhibit capacitation indirectly by diminishing the conformational freedom and hence the biological activity of sperm surface proteins. Alternatively, cholesterol might directly affect specific membrane proteins that function in transmembrane signaling. As shown in Figure 1, either or both of these effects of cholesterol could modulate ion transporters and effector enzymes like sAC. This story, however, is likely to be more complex and to involve additional lipid and sterol species, particularly in light of evidence that phospholipids move between distinct sperm membrane subdomains during capacitation.

Roles of sperm membrane subdomains in signaling In all species examined thus far by visualization of FSCs the plasma membrane overlying the acrosome has been found to be markedly enriched in sterols, relative to either the post-acrosomal plasma membrane or the acrosomal or nuclear membranes (21-24) (Figure 2). The highly conserved demarcation of these two subdomains in the plasma membrane of the sperm head suggests their importance in the organization or control of signal transduction or cellular metabolism. Membrane subdomains enriched in cholesterol and sphingolipids, as opposed to phospholipids, have been suggested to perform these functions in somatic cells. These domains have been termed "membrane rafts," as they are believed to represent liquid-ordered domains in a "sea" of liquiddisordered membrane (refs. 25, 26; see also Simons and Ehehalt, this Perspective series, ref. 27). Their lipid composition makes the raft fractions of membranes separable from nonraft fractions based on their insolubility in detergents such as Triton X-100 or on their lighter buoyant density when centrifuged through density gradients. Using these two biochemical criteria, we have recently demonstrated that mammalian sperm possess such membrane rafts (14).

What then are the functions of cholesterol and these rafts in sperm membranes? The first potential role is in compartmentalizing pathways to specific regions of the cell. This "prefabricated" ordering of pathway components is critical in sperm because of their extraordinarily polarized design, as well as the fact that they are both transcriptionally and translationally inactive. Sperm
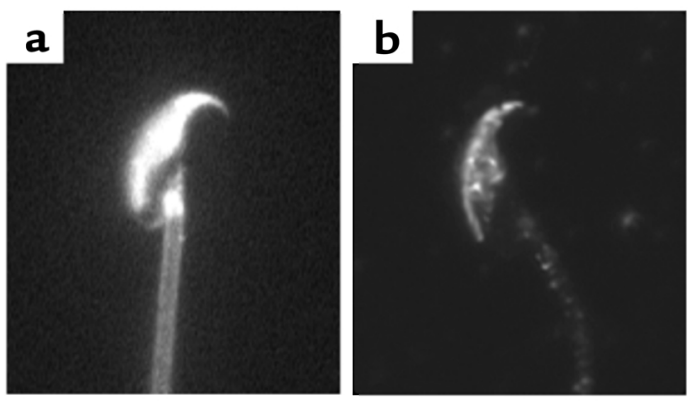

\section{Figure 2}

Localization of sterols and caveolin-1 in noncapacitated murine sperm. (a) The localization of sterols in murine sperm, as visualized by the inherent fluorescence properties of filipin. (b) Immunolocalization of caveolin1 in murine sperm. As performed in ref. 14. 
must assemble and organize their pathways so that they may function precisely where needed, as they cannot synthesize new proteins to meet changing needs in the female tract. One protein enriched in sperm membrane rafts that might function to compartmentalize pathways is caveolin-1 (14). In somatic cells, this protein has been suggested to anchor a variety of signaling and metabolic proteins to membrane rafts (28-30). By scaffolding such molecules, caveolins have been suggested to tether pathway components in "preassembled complexes" that then can be activated by the dissociation of the interaction between the proteins and caveolin (31). Although specific caveolin-interacting proteins have yet to be identified in sperm, we have localized caveolin to the same regions of the sperm that are enriched in cholesterol (Figure 2) and have determined that proteins believed to be important for acrosomal exocytosis are enriched in membrane raft fractions (ref. 14; A.J. Travis et al., unpublished data). Together, these data suggest a role for membrane rafts and caveolin in mediating the localization and/or organization of specific signaling pathways in sperm.

In addition to physically compartmentalizing specific pathways, membrane rafts may regulate such pathways by facilitating the efflux of cholesterol from the sperm plasma membrane. Cholesterol efflux from rafts might initiate signaling by at least two mechanisms. First, efflux could increase membrane fluidity and thus allow previously partitioned integral membrane proteins or membrane-anchored proteins to interact with one another in order to initiate signaling. In this regard, proteins believed to be important in the fusion of the sperm with the egg plasma membrane have been shown to translocate from the anterior to the posterior sperm head during capacitation, suggesting that the loss of cholesterol and the concomitant increase in membrane fluidity is essential for fertilization (32). Second, cholesterol efflux could activate signaling by disrupting the interaction of caveolin with specific signaling molecules, thereby freeing them to form functional signaling complexes. One argument against this latter possibility being critical to sperm function is the finding that mice carrying a targeted deletion of the Caveolin 1 gene appear to be fertile $(33,34)$. However, given the essential role of sperm in the propagation of life, a redundancy of systems would not be unexpected. Indeed, knockout models of several genes hypothesized to be critical for fertilization have resulted in only subtle reductions in male fertility $(35,36)$.

If sperm membrane rafts function in part by mediating cholesterol efflux, then a loss of cholesterol from such regions should be observed when sperm are incubated under capacitating conditions. In fact, cholesterol efflux results in dramatic changes in the pattern and number of FSCs, with loss from the plasma membrane overlying the acrosome, some diffusion of FSCs into the post-acrosomal plasma membrane, and loss from the plasma membrane of the flagellum $(21,23,24)$. A semiquantitative analysis of cholesterol efflux, based on the density of FSCs in different sperm regions before and after capacitation, suggests that efflux occurs from all areas of the sperm that originally contained cholesterol, including both the head and the flagellum (24). Hence, the molecule or molecules that mediate this efflux are likely widespread throughout this cell.

\section{Models of cholesterol efflux from sperm membranes}

In somatic cells, several molecular pathways have been proposed to carry out cholesterol efflux (see Tall et al., this Perspective series, ref. 37). These include unmediated aqueous diffusion, interactions with lipid-poor apolipoproteins, membrane micro-solubilization, or efflux that is mediated by specific molecules by either facilitated or active transport mechanisms $(38,39)$. Molecules proposed to mediate these processes include the scavenger receptors SR-BI and CD36 (40), members of the ATP-binding cassette (ABC) transporter family (41), and caveolin (42), although the role of this latter candidate has been controversial (43). Several of these proteins function most efficiently in concert with a specific class of sterol acceptor. For example, SR-BI mediates efflux to HDL, whereas ABC-A1 mediates efflux to lipid-poor apolipoproteins such as apoA1. Because simple diffusion into an aqueous medium is inefficient, physiological cholesterol efflux from sperm most likely is enhanced by the presence of cholesterol acceptors in the luminal fluid of the female tract. As mentioned above, this phenomenon can be mimicked in vitro by incubating sperm in the presence of a cholesterol acceptor such as serum albumin or $\beta$-cyclodextrins.

Several reports suggest that the cholesterol acceptors HDL and albumin, which are both found in oviductal and follicular fluid, can stimulate capacitation $(44,45)$. Interestingly, human follicular fluid albumin provides a more efficient sink for cellular cholesterol than does the better described serum albumin (44). Moreover, HDL levels in bovine oviductal fluid vary over the estrous cycle, having an inverse log relationship with serum progesterone (45). Thus, the abundance of a known cholesterol acceptor increases at the time of estrus. A better understanding of the nature of these changes and their regulation could shed light on the mechanism of cholesterol efflux from the sperm plasma membrane and might be of great benefit for helping define specific subsets of idiopathic infertility. Such information might also suggest alternative approaches to contraception. However, knockout mice lacking each of the genes encoding the most obvious candidates mentioned above have been generated and are apparently fertile $(33,34,46,47)$. These negative findings may be explained by the effect of oviductal fluid albumin, which is plentiful and can function as a cholesterol acceptor to activate sperm function. Albumin is believed to function nonspecifically by providing a relatively hydrophobic environment in the vicinity of the plasma membrane, facilitating the otherwise inefficient diffusion of cholesterol into an aqueous medium. Efflux to oviductal fluid albumin might provide a redundant, nonspecific mechanism for efflux in vivo, thus safeguarding sperm signaling and fertilization competence when the specific pathways are compromised, as in the knockout models studied. 
Recent work on phospholipid scramblases has begun to clarify the relationship between $\mathrm{HCO}_{3}{ }^{-}$and $\mathrm{Ca}^{2+}$ signaling and the induction of cholesterol efflux during capacitation $(48,49)$. These enzymes translocate choline phospholipids to the inner leaflet, and aminophospholipids to the outer leaflet along their concentration gradients, thus reducing the asymmetry of phospholipid distribution across the membrane bilayer (50). Phospholipid scrambling in sperm appears to require both exposure to $\mathrm{HCO}_{3}{ }^{-}$and PKA activity $(48,51-53)$, and it has been proposed that cAMP generated by sAC triggers a downstream increase in phospholipid scramblase activity, which in turn facilitates cholesterol efflux, potentially through a mediator such as SR-BI (53). A variation of this model, reflecting our observation that rafts are dissipated during capacitation, is shown in Figure 1. Either caveolin or the local topography of a membrane subdomain such as a raft might also promote efflux by providing a clustering of cholesterol, raising its local concentration. This would, in turn, promote efflux down a gradient either specifically through SR-BI to HDL, or nonspecifically to albumin.

\section{Other sterols and lipids in the sperm}

Despite the focus on cholesterol above, it should be noted that sperm are remarkable for the variety of sterols they possess. The sperm of rodents, primates, and other species contain varying amounts of desmosterol $(21,54)$, which undergoes efflux from the sperm membrane during capacitation and could function in a manner similar to that of cholesterol (21). Sperm cells of different species also contain variable amounts of sterol sulfates $(10,21)$. Sterol sulfotransferases, which have been reported to exist within the female tract, are presumed to render sperm membranes more fluid as part of capacitation (55).

Ceramides, another class of membrane lipids that has been implicated in cell signaling, may also contribute to the control of sperm function. For example, increasing sperm ceramide levels has been shown to enhance capacitation by increasing the efflux of cholesterol and desmosterol (56). However, it is unclear whether this effect is through the direct action of the ceramide produced on downstream signaling proteins, or through an increase in lipid disorder, such as that promoted by the phospholipid scramblases. The basic structural component of sphingolipids, ceramide, can be formed by the degradation of sphingomyelin by sphingomyelinase. As recently reviewed by Kolesnick (57), ceramide can exert signaling effects on cells via several independent mechanisms. First, it can increase membrane fluidity by changing lipid packing. In addition, ceramide can directly affect the activity of protein phosphatases and protein kinases. Finally, ceramide can act indirectly by its degradation via ceramidase into sphingosine, which can be phosphorylated by sphingosine kinase into sphingosine-1-phosphate (S1P). This highly reactive compound has been shown to stimulate a $\mathrm{G}$ protein-coupled receptor, $\mathrm{S}_{\mathrm{P}} \mathrm{P}_{1}$ or EDG-1 (58). Given the complex and dynamic sterol and lipid composition of sperm, much work needs to be done to elucidate the pathways regulating sterol/lipid efflux and transducing such efflux into downstream signaling events that ultimately regulate sperm function.

\section{Conclusions and future directions}

We are now in a position to make significant advances in our understanding of sperm capacitation at the molecular level, progress that should ultimately lead to new and better techniques for enhancing fertility, identifying and treating certain forms of male infertility, and preventing conception. One remarkable insight from this work has been the importance of membrane cholesterol efflux in initiating transmembrane signaling events that confer fertilization competence. The identity of the physiologically relevant cholesterol acceptors and modulators of cholesterol efflux is therefore of great interest. Still, it is clear that cholesterol efflux represents only a part of this story. The involvement of phospholipid translocation in mediating dynamic changes in the membrane, rendering it conducive to transmembrane signaling, and the modulation of membrane components of signal transduction cascades by cholesterol or phospholipids will yield important insights into the links between environmental sensing and transmembrane signaling in the sperm. How these dynamic events within the membrane are integrated with membrane microdomains and rafts should provide new and exciting avenues of investigation for the foreseeable future.

\section{Acknowledgments}

This work was supported by NIH grants $1 \mathrm{~K} 01$ RR00188 (to A.J. Travis) and P01-HD06274 (to G.S. Kopf) and a CONRAD Mellon Junior Investigator Award (to A.J. Travis).

1. Austin, C.R. 1952. The "capacitation" of the mammalian sperm. Nature. 170:326.

2. Chang, M.C. 1951. Fertilizing capacity of spermatozoa deposited into the fallopian tubes. Nature. 168:697-698.

3. Wassarman, P.M. 1999. Mammalian fertilization: molecular aspects of gamete adhesion, exocytosis, and fusion. Cell. 96:175-183.

4. Kopf, G.S. 2002. Signal transduction mechanisms regulating sperm acrosomal exocytosis. In Fertilization. D.M. Hardy, editor. Academic Press Inc. San Diego, California, USA. 181-223.

5. Quill, T.A., and Garbers, D.L. 2002. Sperm motility activation and chemoattraction. In Fertilization. D.M. Hardy, editor. Academic Press Inc. San Diego, California, USA. 29-55.

6. Suarez, S.S. 1998. The oviductal sperm reservoir in mammals: mechanisms of formation. Biol. Reprod. 58:1105-1107.

7. Kopf, G.S., Visconti, P.E., and Galantino-Homer, H. 1999. Capacitation of the mammalian spermatozoon. Advances in Developmental Biochemistry. 5:81-105.

8. Jaiswal, B.S., and Eisenbach, M. 2002. Capacitation. In Fertilization. D.M. Hardy, editor. Academic Press Inc. San Diego, California, USA. 57-117.

9. Yanagimachi, R. 1994. Mammalian fertilization. In The physiology of reproduction. E. Knobil and J.D. Neill, editors. Raven Press. New York, New York, USA. 189-317.

10. Cross, N.L. 1998. Role of cholesterol in sperm capacitation. Biol. Reprod. 59:7-11.

11. Visconti, P.E., et al. 1995. Capacitation of mouse spermatozoa. I. Correlation between the capacitation state and protein tyrosine phosphorylation. Development. 121:1129-1137.

12. Hamilton, D.W., Jones, A.L., and Fawcett, D.W. 1969. Cholesterol biosynthesis in the mouse epididymis and ductus deferens: a biochemical and morphological study. Biol. Reprod. 1:167-184.

13. Scott, T.W. 1973. Lipid metabolism of spermatozoa. J. Reprod. Fertil. Suppl. 18:65-76.

14. Travis, A.J., et al. 2001. Expression and localization of caveolin-1, and the presence of membrane rafts, in mouse and Guinea pig spermatozoa. Dev. Biol. 240:599-610.

15. Mittag, T.W., Guo, W.B., and Kobayashi, K. 1993. Bicarbonate-activated 
adenylyl cyclase in fluid-transporting tissues. Am. J. Physiol. 264:F1060-F1064.

16. Buck, J., Sinclair, M.L., Schapal, L., Cann, M.J., and Levin, L.R. 1999. Cytosolic adenylyl cyclase defines a unique signaling molecule in mammals. Proc. Natl. Acad. Sci. USA. 96:79-84.

17. Chen, Y., et al. 2000. Soluble adenylyl cyclase as an evolutionarily conserved bicarbonate sensor. Science. 289:625-628.

18. Jaiswal, B.S., and Conti, M. 2001. Identification and functional analysis of splice variants of the germ cell soluble adenylyl cyclase. J. Biol. Chem. 276:31698-31708.

19. Langlais, J., and Roberts, K.D. 1985. A molecular membrane model of sperm capacitation and the acrosome reaction of mammalian spermatozoa. Gamete Res. 12:183-224.

20. Visconti, P.E., et al. 1999. Cholesterol efflux-mediated signal transduction in mammalian sperm. $\beta$-Cyclodextrins initiate transmembrane signaling leading to an increase in protein tyrosine phosphorylation and capacitation. J. Biol. Chem. 274:3235-3242.

21. Visconti, P.E., et al. 1999. Cholesterol efflux-mediated signal transduction in mammalian sperm: cholesterol release signals an increase in protein tyrosine phosphorylation during mouse sperm capacitation. Dev. Biol. 214:429-443.

22. Friend, D.S. 1982. Plasma-membrane diversity in a highly polarized cell. J. Cell Biol. 93:243-249.

23. Suzuki, F. 1988. Changes in the distribution of intramembranous particles and filipin-sterol complexes during epididymal maturation of golden hamster spermatozoa. J. Ultrastruct. Mol. Struct. Res. 100:39-54.

24. Lin, Y., and Kan, F.W. 1996. Regionalization and redistribution of membrane phospholipids and cholesterol in mouse spermatozoa during in vitro capacitation. Biol. Reprod. 55:1133-1146.

25. Smart, E.J., et al. 1999. Caveolins, liquid-ordered domains, and signal transduction. Mol. Cell. Biol. 19:7289-7304.

26. Simons, K., and Toomre, D. 2000. Lipid rafts and signal transduction. Nat. Rev. Mol. Cell Biol. 1:31-39.

27. Simons, K., and Ehehalt, R. 2002. Cholesterol, lipid rafts, and disease. J. Clin. Invest. 110:597-603. doi:10.1172/JCI200216390.

28. Razani, B., Rubin, C.S., and Lisanti, M.P. 1999. Regulation of cAMP-mediated signal transduction via interaction of caveolins with the catalytic subunit of protein kinase A. J. Biol. Chem. 274:26353-26360.

29. Li, S., et al. 1995. Evidence for a regulated interaction between heterotrimeric G proteins and caveolin. J. Biol. Chem. 270:15693-15701.

30. Scherer, P.E., and Lisanti, M.P. 1997. Association of phosphofructokinase$\mathrm{M}$ with caveolin-3 in differentiated skeletal myotubes. Dynamic regulation by extracellular glucose and intracellular metabolites. J. Biol. Chem. 272:20698-20705.

31. Okamoto, T., Schlegel, A., Scherer, P.E., and Lisanti, M.P. 1998. Caveolins, a family of scaffolding proteins for organizing "preassembled signaling complexes" at the plasma membrane. J. Biol. Chem. 273:5419-5422.

32. Cowan, A.E., Koppel, D.E., Vargas, L.A., and Hunnicutt, G.R. 2001. Guinea pig fertilin exhibits restricted lateral mobility in epididymal sperm and becomes freely diffusing during capacitation. Dev. Biol. 236:502-509.

33. Drab, M., et al. 2001. Loss of caveolae, vascular dysfunction, and pulmonary defects in caveolin-1 gene-disrupted mice. Science. 293:2449-2452.

34. Razani, B., et al. 2001. Caveolin-1 null mice are viable but show evidence of hyperproliferative and vascular abnormalities. J. Biol. Chem. 276:38121-38138.

35. Baba, T., Azuma, S., Kashiwabara, S., and Toyoda, Y. 1994. Sperm from mice carrying a targeted mutation of the acrosin gene can penetrate the oocyte zona pellucida and effect fertilization. J. Biol. Chem. 269:31845-31849.

36. Lu, Q., and Shur, B.D. 1997. Sperm from beta 1,4-galactosyltransferasenull mice are refractory to $\mathrm{ZP} 3$-induced acrosome reactions and penetrate the zona pellucida poorly. Development. 124:4121-4131.

37. Tall, A.R., Costet, P., and Wang, N. 2002. Regulation and mechanisms of macrophage cholesterol efflux. J. Clin. Invest. In press. doi:10.1172/ JCI200216391.

38. Rothblat, G.H., et al. 1999. Cell cholesterol efflux: integration of old and new observations provides new insights. J. Lipid Res. 40:781-796.

39. Fielding, C.J., and Fielding, P.E. 2001. Cellular cholesterol efflux. Biochim. Biophys. Acta. 1533:175-189.

40. Krieger, M. 1998. The "best" of cholesterols, the "worst" of cholesterols: a tale of two receptors. Proc. Natl. Acad. Sci. USA. 95:4077-4080.

41. Santamarina-Fojo, S., Remaley, A.T., Neufeld, E.B., and Brewer, H.B., Jr 2001. Regulation and intracellular trafficking of the ABCA1 transporter J. Lipid Res. 42:1339-1345.

42. Fielding, P.E., and Fielding, C.J. 1995. Plasma membrane caveolae mediate the efflux of cellular free cholesterol. Biochemistry. 34:14288-14292.

43. Matveev, S., Uittenbogaard, A., van Der Westhuyzen, D., and Smart, E.J. 2001. Caveolin-1 negatively regulates SR-BI mediated selective uptake of high-density lipoprotein-derived cholesteryl ester. Eur. J. Biochem. 268:5609-5616.

44. Langlais, J., et al. 1988. Identification of sterol acceptors that stimulate cholesterol efflux from human spermatozoa during in vitro capacitation. Gamete Res. 20:185-201.

45. Ehrenwald, E., Foote, R.H., and Parks, J.E. 1990. Bovine oviductal fluid components and their potential role in sperm cholesterol efflux. Mol. Reprod. Dev. 25:195-204.

46. Febbraio, M., et al. 1999. A null mutation in murine CD36 reveals an important role in fatty acid and lipoprotein metabolism. J. Biol. Chem. 274:19055-19062.

47. McNeish, J., et al. 2000. High density lipoprotein deficiency and foam cell accumulation in mice with targeted disruption of ATP-binding cassette transporter-1. Proc. Natl. Acad. Sci. USA. 97:4245-4250.

48. Harrison, R.A.P., Ashworth, P.J.C., and Miller, N.G.A. 1996. Bicarbonate/CO2, an effector of capacitation, induces a rapid and reversible change in the lipid architecture of boar sperm plasma membranes. Mol. Reprod. Dev. 45:378-391.

49. Gadella, B.M., Miller, N.G., Colenbrander, B., van Golde, L.M., and Harrison, R.A. 1999. Flow cytometric detection of transbilayer movement of fluorescent phospholipid analogues across the boar sperm plasma membrane: elimination of labeling artifacts. Mol. Reprod. Dev. 53:108-125.

50. Basse, F., Stout, J.G., Sims, P.J., and Wiedmer, T. 1996. Isolation of an erythrocyte membrane protein that mediates $\mathrm{Ca} 2+$-dependent transbilayer movement of phospholipid. J. Biol. Chem. 271:17205-17210.

51. Purohit, S.B., Laloraya, M., and Kumar, P.G. 1998. Bicarbonate-dependent lipid ordering and protein aggregation are part of the nongenomic action of progesterone on capacitated spermatozoa. J. Androl. 19:608-618.

52. Gadella, B.M., and Harrison, R.A. 2000. The capacitating agent bicarbonate induces protein kinase A-dependent changes in phospholipid transbilayer behavior in the sperm plasma membrane. Development. 127:2407-2420

53. Flesch, F.M., et al. 2001. Bicarbonate stimulated phospholipid scrambling induces cholesterol redistribution and enables cholesterol depletion in the sperm plasma membrane. J. Cell Sci. 114:3543-3555.

54. Lin, D.S., Connor, W.E., Wolf, D.P., Neuringer, M., and Hachey, D.L. 1993. Unique lipids of primate spermatozoa: desmosterol and docosahexaenoic acid. J Lipid Res. 34:491-499.

55. Langlais, J., et al. 1981. Localization of cholesteryl sulfate in human spermatozoa in support of a hypothesis for the mechanism of capacitation. Proc. Natl. Acad. Sci. USA. 78:7266-7270.

56. Cross, N.L. 2000. Sphingomyelin modulates capacitation of human sperm in vitro. Biol. Reprod. 63:1129-1134.

57. Kolesnick, R. 2002. The therapeutic potential of modulating the ceramide/sphingomyelin pathway. J. Clin. Invest. 110:3-8. doi:10.1172/ JCI200216127.

58. Hla, T., Lee, M.J., Ancellin, N., Paik, J.H., and Kluk, M.J. 2001. Lysophospholipids: receptor revelations. Science. 294:1875-1878 